\title{
Anti-endothelial cell antibodies do not correlate with disease activity in systemic sclerosis
}

\author{
Małgorzata Michalska-Jakubus, Małgorzata Kowal, Michat Adamczyk, Dorota Krasowska
}

Department of Dermatology, Venereology and Pediatric Dermatology, Medical University of Lublin, Lublin, Poland

Adv Dermatol Allergol 2018; XXXV (2): 185-191

DOI: https://doi.org/10.5114/ada.2018.75241

\begin{abstract}
Introduction: Anti-endothelial cell antibodies (AECA) recognize endothelial cell proteins and are thought to play an important role in vascular damage observed in systemic scleroderma (SSc) and many other autoimmune diseases. In SSc, AECA were found to be more common in patients with pulmonary hypertension, digital ulcers and nailfold capillaroscopic changes. Until now, there have been no studies examining the association between AECA positivity with the activity and duration of the disease.

Aim: To evaluate associations between the presence of AECA in sera of patients with SSC and internal organs involvement as well as disease activity.

Material and methods: Sera of 58 patients with SSc (50 with localized subtype and 8 with diffuse subtype) were examined for AECA presence using an indirect immunofluorescence technique. Several clinical and laboratory features were also evaluated as well as disease activity and disease duration.

Results: A significant association between positive AECA and a subtype of SSc $(p=0.021)$ was found, as well as between presence of digital ulcers and digital scars $(p=0.001)$, calcinosis $(p=0.02)$, acroosteolysis $(p=0.028)$ and a nearly significant association between AECA and lung fibrosis $(p=0.47)$. No association between disease duration, disease activity and AECA ( $p=1.000$ and 0.191 , respectively) was present.

Conclusions: Anti-endothelial cell antibodies are not associated with the activity of SSc. Digital ulcers, calcinosis and acroosteolysis are more common among AECA-positive patients suggesting that the presence of AECA might be an indicator of vascular complications development in SSc. Positive AECA among patients with lung fibrosis indicate their possible role in the development of lung disease. Further prospective studies including a greater number of patients are required.
\end{abstract}

Key words: anti-endothelial cell antibodies, digital ulcers, indirect immunofluorescence, lung fibrosis, systemic scleroderma, vascular damage.

\section{Introduction}

Systemic scleroderma (SSC) is an autoimmune connective tissue disease (CTD) in which microvascular injury plays an important role. Vascular damage is accompanied by activation of the immune system and fibroblasts, which results in autoantibodies production, inflammation and excessive collagen deposition in the skin and internal organs, however the pathogenesis of SSc is still not completely understood. Endothelial cells (ECS) damage is thought to be the very early stage of pathologic processes involved in the development of SSc. Several mechanisms are suggested to be responsible, including defective nitric oxide production, overexpression of
$V_{E G F_{165}} b$ and increased oxidative stress with overproduction of reactive oxygen species. Furthermore, in SSc decreased amounts of circulating EC progenitor cells were found in peripheral blood, which may be associated with impaired regeneration of endothelium. Several immunological mechanisms have also been postulated, including cytotoxic T lymphocytes activity, ECs apoptosis induced by neutrophils, activation of the classical complement pathway, and last but not least anti-endothelial cell antibodies (AECA) activity [1, 2].

AECA activity are a heterogeneous group of autoantibodies that recognize ECs proteins and particles present on the ECs surface. AECA activity have been described in a number of autoimmune diseases and other

Address for correspondence: Michał Adamczyk MD, Department of Dermatology, Venereology and Pediatric Dermatology, Medical University of Lublin, 13 Radziwiłłowska St, 20-080 Lublin, Poland, phone/fax: +48 815323 647, mobile: +48 693 522 384 , e-mail: michaladamczyk1310@wp.pl Received: 7.11.2016, accepted: 1.02.2017. 
conditions [3]. Data concerning prevalence of AECA in SSc patients present highly variable results (22-86\%), which probably results from diversity of study populations and detection techniques [4].

Many pathologic mechanisms of AECA in SSc are considered, including EC activation, induction of coagulation, AECA-induced cytotoxicity and apoptosis of ECs, as well as activation of fibroblasts [4]. Still there are concerns whether AECA are just an epiphenomenon of vascular injury without pathogenicity or they underlie SSc-related microangiopathy. Literature data concerning associations of AECA and clinical features of SSC are quite poor. AECA activity were reported to be more common in patients with digital ulcers and gangrene [5-7], nailfold capillaroscopic changes $[5,6]$ and pulmonary hypertension (PAH) $[6,7]$. Until now, there have been no studies correlating AECA positivity with activity and duration of the disease.

\section{Aim}

The aim of the study was to evaluate associations between the presence of AECA in sera of patients with SSc and internal organs involvement as well as disease activity.

\section{Material and methods}

\section{Study population}

The study comprised 58 consecutive SSc patients (mean age: 56.57 years, range: $25-78$ years) hospitalized in the Department of Dermatology, Venerology and Pediatric Dermatology of Lublin between January 2013 and December 2014. We included all patients, who fulfilled the American Rheumatism Association diagnostic criteria for SSc [8]. Exclusion criteria included: concomitant infections, neoplasms, cardiovascular and pulmonary diseases not associated with SSc as well as cigarette smoking. Ethical approval was obtained from the Bioethics Committee of Medical University of Lublin and each patient signed informed consent before participating in the study.

Study population was divided into two groups according to the disease subtype [9]: limited cutaneous SSc - IcSSc $(n=50)$ and diffuse cutaneous SSc - dcSSc $(n=8)$. Furthermore, based on disease duration from the first non-Raynaud symptom, we divided SSc patients into an early ( $<5$ years for IcSSc and $<3$ years for dcSSc) and late ( $>5$ years for lcSSc and $>3$ years for dcSSc) stage of SSc ( $n=15$ and $n=43$, respectively) [10].

\section{Clinical assessment}

Each patient was carefully examined for the presence of digital scars or ulcers. Modified Rodnan Skin Score was calculated for all studied subjects to determine the severity of skin involvement [11, 12]. Routine laboratory and imaging diagnostic tests were performed to determine dis- ease activity and internal organ involvement. Among laboratory tests we selected serum levels of C-reactive protein (CRP), erythrocyte sedimentation rate (ESR), complement components 3 and $4(C 3, C 4)$ for further analysis.

Lung involvement was evaluated by high resolution computed tomography (HRCT), spirometry and diffusing lung capacity for carbon monoxide (DLCO). In spirometry, performed by BodyScreen II (Jaeger), forced vital capacity (FVC) and total lung capacity (TLC) were analyzed. According to the American Thoracic Society, TLC $\geq 80 \%$ and FVC $\geq 75 \%$ of predicted values were considered normal. TLC $<80 \%$ and FVC $<75 \%$ were interpreted as restriction [13]. Diffusing lung capacity for carbon monoxide was performed by Lung Test 1000 spirometer (MES Ltd.). According to the European Respiratory Society, results $<80 \%$ of predicted values were considered as decreased diffusing lung capacity [14].

Heart function was evaluated by echocardiography (B-mode and color Doppler imagining) and standard, 12-lead electrocardiography (ECG). Esophageal disorders were examined by upper gastrointestinal $\mathrm{X}$-ray series. $\mathrm{X}$ ray images of hands were performed to determine the presence of acroosteolysis and calcinosis.

In order to assess cutaneous microcirculation, nailfold capillaroscopy (NC) was performed in each subject. We used a video-capillaroscope VideoCap 3.0 Derma (DS Medica). According to Cutolo et al., obtained capillaroscopic images were divided into early, active and late patterns [15].

To determine activity of SSc, we used 10-points activity index for SSc developed by the European Scleroderma Study Group (ESCSG). Results $\geq 3$ points were indicative for active disease [16].

\section{Anti-endothelial cell antibodies activity identification}

To measure the presence of AECA, clotted blood samples were collected from all SSc patients in the amount of $5 \mathrm{ml}$. Hyperlipidemic or hemolyzed samples were not analyzed. An indirect immunofluorescence procedure with human umbilical vein endothelial cells (HUVEC) and monkey skeletal muscles as substrates was performed. We used TITERPLANE technique elaborated by EUROIMMUN.

Human umbilical vein endothelial cells cultures and frozen monkey skeletal muscles were incubated for 30 min with diluted $1: 100$ sera from SSc patients on biochip platforms. After incubation, platforms were rinsed with PBSTween wash buffer. During the second stage of incubation AECA antibodies bound to the substrate were identified by goat anti-human IgG antibodies marked with fluorescein (30 min in room temperature), and the rinsed again with PBS-Tween wash buffer. Microscopic evaluation was performed by fluorescent microscope Nikon TS100. Positive reaction was interpreted qualitatively and on ECs was characterized by cytoplasmic, granular, yellowish-green fluorescence concentrated around nucleus (Figure 1). 


\section{Statistical analysis}

Statistical analysis was performed with the use of Statistica 10.0 PL package. Data were analyzed for normal distribution using the Shapiro-Wilk test. For normally distributed data, parametric $t$-Student test was used, while equality of variances was checked by exact Fisher's test. For non-parametric data U Mann-Whitney test was performed. $P$-value $<0.05$ was considered statistically significant.

\section{Results}

AECA activity antibodies were present in 21 patients with SSC (36.21\%). Majority of dcSSc patients were AECApositive $(75 \%, n=6)$, while prevalence of AECA positive individuals among ICSSc patients was 30\% ( $n=15)$. Statistical analysis (Fisher's exact test) revealed a significant association between positive AECA and a subtype of SSC ( $p=0.021$; Table 1). We found no association between disease duration, disease activity and AECA presence $(p=1.000$ and $p=0.191$, respectively).
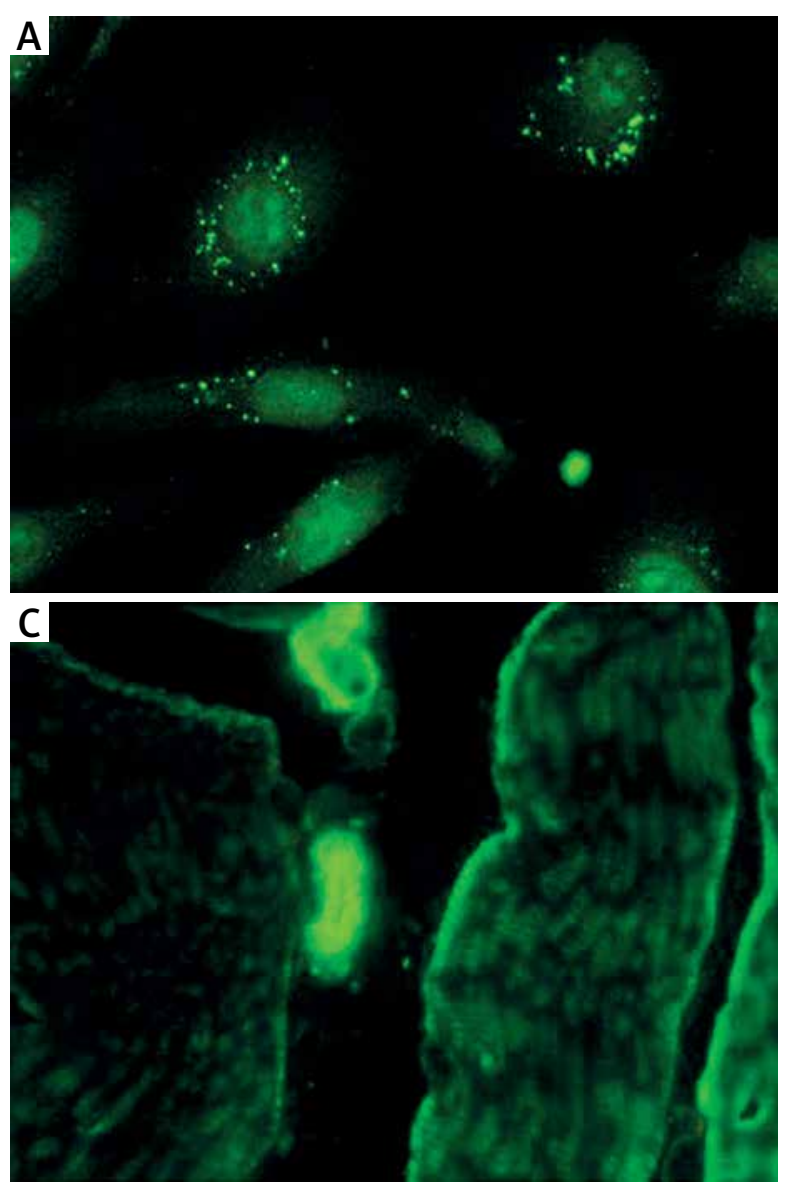

Figure 1. Positive AECA reaction manifesting with cytoplasmic, granular, yellowish-green fluorescence concentrated around nuclei
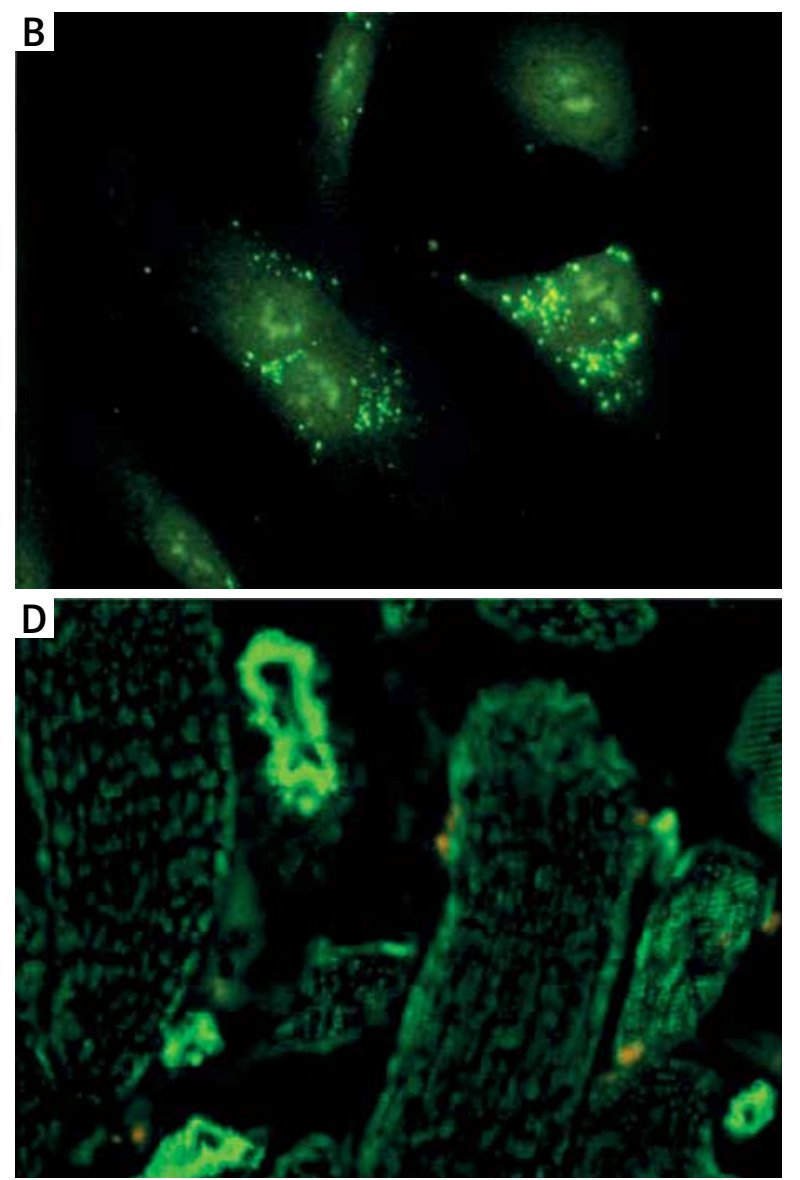

Prevalence of clinical signs, visceral involvement and abnormal clinical test results in patients with SSc are shown in Table 2. In the studied group, we found a significant association between positive AECA and the presence of active digital ulcers or digital scars. AECA were more common in patients with digital scars and active digital ulcers when compared to those without trophic lesions $(p=0.001)$. Furthermore, AECA were significantly more frequent in patients with calcinosis and acroosteolysis (Table 2).

No associations were found between AECA presence and modified Rodnan Skin Score, ECG changes, echocardiographic alterations, esophageal dysfunction and nailfold capillaroscopic pattern. Moreover, alterations in spirometry, DLCO, CRP and ESR elevation, complement components $\mathrm{C} 3$ and $\mathrm{C} 4$ decrease were not more common in patients positive for AECA.

\section{Discussion}

Nearly 45 years have passed since AECA were first detected by Lindquist and Osterland [17]. The presence

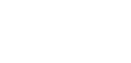

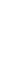

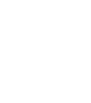


Table 1. Presence of AECA antibodies depending on the SSc subtype, disease duration and disease activity (statistically significant values in bold)

\begin{tabular}{lccccc}
\hline Parameter & & Total & AECA absent & AECA present & \multicolumn{1}{c}{$P$-value } \\
\hline Disease subtype & IcSSc & 50 & $35(70.00 \%)$ & $15(30.00 \%)$ & \multirow{2}{0}{0.021} \\
\cline { 2 - 5 } & dcSSc & 8 & $2(25.00 \%)$ & $6(75.00 \%)$ & \\
\hline \multirow{2}{*}{ Disease duration } & Early & 15 & $10(66.67 \%)$ & $5(33.33 \%)$ & \multirow{2}{*}{1.000} \\
\cline { 2 - 5 } & Late & 43 & $27(62.79 \%)$ & $16(37.21 \%)$ & \\
\hline Disease activity & Inactive & 45 & $31(68.89 \%)$ & $14(31.11 \%)$ & 0.191 \\
\hline
\end{tabular}

Table 2. Organ involvement and laboratory test results in patients with SSc in relation to AECA presence (statistically significant values in bold)

\begin{tabular}{|c|c|c|c|c|c|}
\hline Clinical sign/test result & Present (+)/absent (-) & Total & AECA (-) & $\operatorname{AECA~(+)~}$ & $P$-value \\
\hline \multirow[t]{2}{*}{ Digital ulcers/ scars } & + & 39 & $19(48.72 \%)$ & $20(51.28 \%)$ & 0.001 \\
\hline & - & 19 & $18(94.74 \%)$ & $1(5.26 \%)$ & \\
\hline \multirow[t]{2}{*}{ Calcinosis } & + & 6 & $1(16.67 \%)$ & $5(83.33 \%)$ & 0.02 \\
\hline & - & 52 & $36(69.23 \%)$ & $16(30.77 \%)$ & \\
\hline \multirow[t]{2}{*}{ Acroosteolysis } & + & 22 & $10(45.45 \%)$ & $12(54.55 \%)$ & 0.047 \\
\hline & - & 35 & $26(74.29 \%)$ & $19(25.71 \%)$ & \\
\hline \multirow[t]{2}{*}{ Modified Rodnan skin score } & $\geq 14$ & 6 & $2(33.33 \%)$ & $4(66.67 \%)$ & 0.176 \\
\hline & $<14$ & 52 & $35(67.31 \%)$ & $17(32.69 \%)$ & \\
\hline \multirow[t]{2}{*}{ Lung fibrosis in HRCT } & + & 44 & $25(56.82 \%)$ & $19(43.18 \%)$ & 0.062 \\
\hline & - & 14 & $12(85.71 \%)$ & $2(14.29 \%)$ & \\
\hline \multirow[t]{2}{*}{ Restriction on spirometry } & + & 17 & $13(76.47 \%)$ & $4(23.53 \%)$ & 0.129 \\
\hline & - & 33 & $17(51.52 \%)$ & $16(48.48 \%)$ & \\
\hline \multirow[t]{2}{*}{ ECG changes } & + & 18 & $11(61.11 \%)$ & 7 (38.89\%) & 0.777 \\
\hline & - & 40 & $26(65.00 \%)$ & $14(35.00 \%)$ & \\
\hline \multirow[t]{2}{*}{$\mathrm{ECHO}$} & + & 35 & $22(62.86 \%)$ & $13(37.14 \%)$ & 1.000 \\
\hline & - & 23 & $15(65.22 \%)$ & $8(34.78 \%)$ & \\
\hline \multirow[t]{2}{*}{ Esophageal changes } & + & 43 & $27(62.79 \%)$ & $16(37.21 \%)$ & 1.000 \\
\hline & - & 15 & $10(66.67 \%)$ & $5(33.33 \%)$ & \\
\hline \multirow{3}{*}{$\begin{array}{l}\text { Capillaroscopic pattern } \\
\text { (by Cutolo) }\end{array}$} & Early & 17 & $12(70.59 \%)$ & $5(29.41 \%)$ & $0.625^{c}$ \\
\hline & Active & 14 & $8(57.14 \%)$ & $6(42.86 \%)$ & \\
\hline & Late & 23 & $13(56.52 \%)$ & $10(43.48 \%)$ & \\
\hline \multirow[t]{2}{*}{ DLCO } & Decreased $<80 \%$ & 24 & $18(75.00 \%)$ & $6(25.00 \%)$ & 0.162 \\
\hline & Normal $\geq 80 \%$ & 30 & $16(53.33 \%)$ & $14(46.67 \%)$ & \\
\hline \multirow[t]{2}{*}{ FVC } & Decreased $<75 \%$ & 3 & $2(66.67 \%)$ & $1(33.33 \%)$ & 1.000 \\
\hline & Normal $\geq 75 \%$ & 47 & $28(59.57 \%)$ & $19(40.43 \%)$ & \\
\hline \multirow[t]{2}{*}{ TLC } & Decreased $<80 \%$ & 17 & $13(76.47 \%)$ & $4(23.53 \%)$ & 0.235 \\
\hline & Normal $\geq 80 \%$ & 38 & $22(57.89 \%)$ & $16(42.11 \%)$ & \\
\hline \multirow[t]{2}{*}{ ESR } & Elevated & 38 & $26(68.42 \%)$ & $12(31.58 \%)$ & 0.392 \\
\hline & Normal & 20 & $11(55.00 \%)$ & $9(45.00 \%)$ & \\
\hline \multirow[t]{2}{*}{ CRP } & Elevated & 19 & $12(63.16 \%)$ & 7 (36.84\%) & 1.000 \\
\hline & Normal & 39 & $25(64.10 \%)$ & $14(35.90 \%)$ & \\
\hline \multirow[t]{2}{*}{$\mathrm{C3}$} & Decreased & 8 & $5(62.50 \%)$ & $3(37.50 \%)$ & 1.000 \\
\hline & Normal & 50 & $32(64.00 \%)$ & $18(36.00 \%)$ & \\
\hline \multirow[t]{2}{*}{ C4 } & Decreased & 3 & $2(66.67 \%)$ & $1(33.33 \%)$ & 1.000 \\
\hline & Normal & 55 & $35(63.64 \%)$ & $20(36.36 \%)$ & \\
\hline
\end{tabular}

Significant differences for $\alpha=0.05$ (two-tailed test). ' $\chi^{2}$ test. HRCT - high resolution computed tomography, ECG - electrocardiography, ECHO - echocardiography, DLCO - diffusing capacity of the lung for carbon monoxide, FVC - forced vital capacity, TLC - total lung capacity, ESR - erythrocyte sedimentation rate, CRP-C-reactive protein, C3-complement component 3, C4-complement component 4. 
of AECA was later described in a number of autoimmune disorders including SSc, vasculitis, systemic lupus erythematosus (SLE), rheumatoid arthritis as well as other conditions, such as diabetes mellitus, atherosclerosis, in heart transplant recipients with vascular rejection [18], and, lately in graft versus host disease [19]. Moreover, in recent years there has been a wide interest in the role of AECA in the development of pulmonary arterial hypertension (PAH), both idiopathic and associated with CTDs [20].

Pathological mechanisms of AECA are not fully understood yet. However, data exist that AECA induce activation of ECS which results in inflammatory cytokines production and increase of adhesion molecules expression. Furthermore, AECA are thought to be responsible for antibody-dependent cellular cytotoxicity (ADCC) against ECS, complement system activation and probably to have procoagulatory effects [4]. It was proved that AECA induce apoptosis of EC progenitor cells in bone marrow in SSc patients, which results in disturbances in endothelial regeneration and healing of vascular lesions [21].

Many different methods are used to detect AECA; however, to date, there has been no standardization for AECA identification [22]. Recent studies have focused on identification of AECA target proteins; however, they have yielded divergent results. Garcia de la Pena-Lefebvre et al. concluded that AECA from patients with dcSSc bind to ECs' topoisomerase-1 independently of the presence of anti-Scl-70 antibodies in the patient serum, and this is a distinguishing feature between IcSSc and dcSSc patients [23]. In the next paper the same authors identified ubiquitous CENP-B as the main target of anti-endothelial cell antibodies in patients with ICSSc [24]. In one of the recent studies, Dib et al. used two-dimensional electrophoresis and immunoblotting with protein extracts of HUVECS and mass spectrometry for detection of AECA targets in SSC and PAH patients, and identified them as lamin $A / C$ and tubulin $\beta$ chain. They found the biggest amount of protein spots in patients with SSC and PAH $(n=110)$, less in SSC patients without PAH $(n=82)$ and the lowest amount in idiopathic PAH patients $(n=37)$. However, the functional significance of these findings remains unclear [20].

Lately, interesting research has been published in which one of AECA targets was identified as ICAM-1. The authors have proved that anti-ICAM AECA stimulate proinflammatory activation of human ECs, which manifests as an increase in reactive oxygen species generation and VCAM-1 expression [18].

In the present study we used II Fas a detection method for AECA, with HUVEC and skeletal muscle of monkey as substrates. As shown in laboratory tests, skeletal muscle provide a wider spectrum of antigens, which increases the sensitivity of the method and in SSc patients, the highest prevalence of AECA was obtained with immunoblot with HUVEC as a substrate [25]. In our study the prevalence of AECA among SSc patients was 36.21\%, which is consistent with previous reports.

Our results showed a significant association between the presence of AECA and subset of the disease. AECA were more common among patients with dcSSc than IcSSc. Literature data concerning the association between SSc subset and AECA are somewhat conflicting. Pignone et al. and Salozhin et al. did not find such association $[6,26]$, whereas Salojin et al. observed AECA in 16 of 36 (44\%) patients with ISSc, and 26 of 31 (83\%) patients with dSSc [5].

Furthermore, we have found, consistently with previous reports, that AECA are significantly more common in patients with digital ulcers or digital gangrene [5-7, 26]. In one of the studies, AECA positive patients with digital infarcts and PAH had significantly higher levels of AECA than other AECA-positive patients [7].

Wusirika et al. reported a positive relationship between lung fibrosis in SSc patients evaluated by HRCT as well as PAH and the presence of AECA measured by IIF. In Western blot analysis they found antibodies binding to proteins in extracts of human EC in all patients in whom there was evidence of pulmonary disease [27]. In a study by Lewandowska et al., AECA were highly prevalent in a subgroup of patients suffering from interstitial pulmonary fibrosis (7 of 15 AECA positive patients had lung fibrosis) [28].

In SSc it is postulated that cytokines produced by activated ECs interact with tissue fibroblasts, increasing their proliferation and differentiation as well as inhibiting their apoptosis, which results in collagen overproduction and fibrosis [4]. In the presented study we also found a tendency towards positive association between AECA and lung fibrosis evaluated by HRCT. Reversely, Pignone et al. found no association between HRCT lesions and AECA among 50 patients with SSc. Similar observations come from the report of Salozhin et al. [6, 29].

Considering lung function tests, Pignone et al. reported a higher prevalence of decreased DLCO values among patients with dcSSC and AECA [6]; however, our results did not show such association. There was also no association with decreased FVC and TLC and we found no data in literature concerning such relationship.

According to the microvascular abnormalities observed by nailfold capillaroscopy (NC), our study did not confirm the association between the presence of AECA and the SSc capillaroscopic patterns (early, active or late) (Table 2). Moreover, we did not find any statistically significant differences in AECA positivity depending on the presence and severity of particular changes in capillary morphology typical for SSc microangiopathy, i.e. megacapillaries, microhemorrhages, neoangiogenesis, capillary loss and avascular areas (data not shown). This is somewhat opposite to previous studies. Pignone et al. as well as Salozhin et al. found an association between NC abnormalities and positive AECA in 
SSc patients $[6,26]$. Salojin et al. reported a relationship between positive AECA and vessels tortuosity of grade 3 [5]. Riccieri et al. found that AECA-positive patients had more severe NC changes, in particular late capillaroscopic pattern [29].

Pignone et al. as well as Salozhin et al. found no relationship between skin involvement measured by Rodnan skin score and positive AECA [6, 26], which is consistent with our results. Reversely, in a study by Riccieri et al., patients with higher AECA serum levels showed significantly greater results of modified skin score $(\geq 15$, $p<0.04$ ) [29]. In our study, the lack of association between AECA presence and modified Rodnan skin score may be a result of a limited sample size for dcSSc.

We were not able to confirm any association between AECA and heart involvement. In the current literature there are only two studies evaluating cardiac disease in SSC in relation to AECA and in both of them, cardiac dysfunction assessed by echocardiography and ECG was not more common in AECA-positive patients $[6,26]$. Moreover, we found a positive association between AECA and acroosteolysis and no relationship between AECA and esophageal involvement, which is consistent with the study by Salozhin et al. [26]. Interestingly, we found a significant association between calcinosis and AECA presence in contrast to other reports [26].

Finally, in some systemic inflammatory conditions, i.e. SLE and systemic vasculitis AECA titers were found to correlate well with disease activity [30, 31]. There are no data in the literature concerning the relationship between SSC activity and the presence of AECA. We found no associations between CRP, ESR, C 3 and C4 serum levels and AECA, and our results did not confirm the relationship between overall activity of SSc measured by EsCSG disease activity index and AECA. We also did not find any association between disease duration and AECA and there are no literature data on that topic.

The limitations of the present study include its single center character, small sample size and a limited number of patients with dcSSc enrolled. Furthermore, patients were recruited consecutively and only female patients were included in the study.

\section{Conclusions}

Anti-endothelial cell antibodies do not correlate with the activity of SSc. Digital ulcers, calcinosis and acroosteolysis are more common among AECA-positive patients suggesting that the presence of AECA might be an indicator of vascular complication development in SSc, although they are not associated with NC patterns. Positive AECA among patients with lung fibrosis indicate their possible role in the development of lung disease. Further prospective studies including a greater number of patients with a unified and standardized detection method are required to fully elucidate the clinical significance of AECA as well as their prognostic value in SSC.

\section{Conflict of interest}

The authors declare no conflict of interest.

\section{References}

1. Altorok N, Wang Y, Kahaleh B. Endothelial dysfunction in systemic sclerosis. Curr Opin Rheumatol 2014; 26: 615-20.

2. Gerlicz-Kowalczuk Z, Dziankowska-Zaborszczyk E, Dziankowska-Bartkowiak B. Circulating angiostatin serum level in patients with systemic sclerosis. Adv Dermatol Allergol 2017; 34: 543-6.

3. Domiciano DS, Carvalho JF, Shoenfeld Y. Pathogenic role of anti endothelial cell antibodies in autoimmune rheumatic diseases. Lupus 2009; 18: 1233-8.

4. Mihai C, Tervaert JW. Anti-endothelial cell antibodies in systemic sclerosis. Ann Rheum Dis 2010; 69: 319-24.

5. Salojin KV, Le Tonqueze M, Saraux A, et al. Antiendothelial cell antibodies: useful markers of systemic sclerosis. Am J Med 1997; 102: 178-85.

6. Pignone A, Scaletti C, Matucci-Cerinic M, et al. Anti-endothelial cell antibodies in systemic sclerosis: significant association with a vascular involvement and alveolocapillary impairment. Clin Exp Rheumatol 1998; 16: 527-32.

7. Negi VS, Tripathy NK, Misra R, Nityanand S. Antiendothelial cell antibodies in scleroderma correlate with severe digital ischemia and pulmonary arterial hypertension. J Rheumatol 1998; 25: 461-5.

8. Preliminary criteria for the classification of systemic sclerosis (scleroderma). Subcommittee for scleroderma criteria of the American Rheumatism Association Diagnostic and Therapeutic Criteria Committee. Arthritis Rheum 1980; 23: 581-90.

9. LeRoy EC, Black C, Fleischmajer R, et al. Scleroderma (systemic sclerosis): classification, subsets and pathogenesis. J Rheumatol 1988; 15: 202-5.

10. Steen VD, Medsger TA. Classification, prognosis. In: Systemic Sclerosis. Clements PJ, Furst D (eds). Williams\&Wilkins, Baltimore 1996; 121-52.

11. Rodnan GP, Lipinski E, Luksick J. Skin thickness and collagen content in progressive systemic sclerosis (scleroderma) and localised scleroderma. Arthritis Rheum 1979; 22: 130-40.

12. Furt DE, Clements PJ, Steen VD, et al. The modified Rodnan skin score is an accurate reflection of skin biopsy thickness in systemic sclerosis. J Rheumatol 1998; 25: 84-8.

13. Gardner RM, Hankinson JL. Standardization of spirometry 1987 ATS update (American Thoracic Society). J Occup Med 1988; 30: 272-3.

14. Miller A, Enright PL. PFT interpretive strategies: American Thoracic Society/ European Respiratory Society 2005 guideline gaps. Respir Care 2012; 57: 127-33.

15. Cutolo M, Sulli A, Pizzorni C, Accardo S. Nailfold videocapillaroscopy assessment of microvascular damage in systemic sclerosis. J Rheumatol 2000; 27: 155-60.

16. Valentini G, Della Rossa A, Bombardieri S, et al. European multicentre study to define disease activity criteria for systemic sclerosis. Identification of disease activity variables and development of preliminary activity indexes. Ann Rheum Dis 2001; 60: 592-8.

17. Lindquist KJ, Osterland CK. Human antibodies to vascular endothelium. Clin Exp Immunol 1971; 9: 753-60. 
18. Wolf SI, Howat S, Abraham DJ, et al. Agonistic anti-ICAM 1 antibodies in scleroderma: activation of endothelial proinflammatory cascades. Vascul Pharmacol 2013; 59: 19-26.

19. Yao J, Song A, Cao W, et al. Clinical significance of anti-endothelial cell antibody in allogeneic hematopoietic stem cell transplantation recipients with graft-versus-host disease. Int J Hematol 2014; 99: 329-37.

20. Dib H, Tamby MC, Bussone G, et al. Targets of anti-endothelial cell antibodies in pulmonary hypertension and scleroderma. Eur Respir J 2012; 39: 1405-14.

21. Del Papa N, Quirici N, Scavullo C, et al. Antiendothelial cell antibodies induce apoptosis of bone marrow endothelial progenitors in systemic sclerosis. J Rheumatol 2010; 37: 2053-63.

22. Belizna C, Cohen-Tervaert JW. Specificity, pathogenecity, and clinical value of antiendothelial cell antibodies. Semin Arthritis Rheum 1997; 27: 98-109.

23. Garcia de la Pena-Lefebvre P, Chanseaud Y, Tamby MC, et al. IgG reactivity with a 100-kDa tissue and endothelial cell antigen identified as topoisomerase I distinguishes between limited and diffuse systemic sclerosis patients. Clin Immunol 2004; 111: 241-51.

24. Servettaz A, Tamby MC, Guilpain P, et al. Anti-endothelial cell antibodies from patients with limited cutaneous systemic sclerosis bind to centromeric protein B (CENP-B). Clin Immunol 2006; 120: 212-9.

25. Renaudineau Y, Revelen R, Levy Y, et al. Anti-endothelial cell antibodies in systemic sclerosis. Clin Diagn Lab Immunol 1999; 6: 156-60.

26. Salozhin KV, Shcherbakov AB, Nasonov EL, et al. Antiendothelial antibodies in systemic scleroderma and Raynaud's disease. Ter Arkh 1995; 67: 54-7.

27. Wusirika R, Ferri C, Marin M, et al. The assessment of antiendothelial cell antibodies in scleroderma-associated pulmonary fibrosis. A study of indirect immunofluorescent and western blot analysis in 49 patients with scleroderma. Am J Clin Pathol 2003; 120: 596-606.

28. Lewandowska K, Ciurzynski M, Gorska E, et al. Antiendothelial cells antibodies in patients with systemic sclerosis in relation to pulmonary hypertension and lung fibrosis. Adv Exp Med Biol 2013; 756: 147-53.

29. Riccieri V, Germano V, Alessandri C, et al. More severe nailfold capillaroscopy findings and anti-endothelial cell antibodies. Are they useful tools for prognostic use in systemic sclerosis? Clin Exp Rheumatol 2008; 26: 992-7.

30. Li JS, Liu MF, Lei HY. Characterization of anti-endothelial cell antibodies in the patients with systemic lupus erythematosus: a potential marker for disease activity. Clin Immunol Immunopathol 1996; 79: 211-6.

31. Park MC, Park YB, Jung SY, et al. Anti-endothelial cell antibodies and antiphospholipid antibodies in Takayasu's arteritis: correlations of their titers and isotype distributions with disease activity. Clin Exp Rheumatol 2006; 24 (2 Suppl 41): S10-6. 